\title{
Smarandache Ruled Surfaces according to Darboux Frame in $E^{3}$
}

\author{
Soukaina Ouarab \\ Hassan II University of Casablanca, Ben M'sik Faculty of Sciences, Department of Mathematics and Computer Science, \\ Casablanca 20000, Morocco \\ Correspondence should be addressed to Soukaina Ouarab; soukaina.ouarab.sma@gmail.com
}

Received 15 March 2021; Revised 25 April 2021; Accepted 13 May 2021; Published 26 May 2021

Academic Editor: Niansheng Tang

Copyright (C) 2021 Soukaina Ouarab. This is an open access article distributed under the Creative Commons Attribution License, which permits unrestricted use, distribution, and reproduction in any medium, provided the original work is properly cited.

This paper presents a new approach of constructing special ruled surfaces and aims to study their developability and minimalist conditions. Our concept opens opportunities for application in engineering, surface modeling, and architectural design. The principle of our study is to introduce the original definitions of Smarandache ruled surfaces according to Darboux frame of a curve lying on an arbitrary regular surface in $E^{3}$. It concerns $T g$-Smarandache ruled surface, $T n$-smarandache ruled surface, and $g n$-Smarandache ruled surface. New theorems giving necessary and sufficient conditions for those surfaces to be developable and minimal are investigated. Finally, an example with illustrations is presented.

\section{Introduction}

In the classical differential geometry $[1,2]$, a ruled surface is generated by a straight line moving along a curve $c(s)$. The various positions of the generating line are called the rulings. Such a surface has the following parametric representation:

$$
\omega:(s, v) \in I \times \mathbb{R} \longmapsto c(s)+v \vec{X}(s),
$$

where $I$ is an open interval of $\mathbb{R}, c(s)$ is a curve through which all rulings pass (it is called the base curve), and $\vec{X}(s)$ are the rulings directors of the ruled surface.

Developability and minimalist notions are two of the most important properties that can be studied for ruled surfaces.

Developable ruled surfaces can be transformed into the plane without any deformation and distortion, and they form relatively small subsets that contain cylinders, cones, and the tangent surfaces. They are characterized with vanishing Gaussian curvature [3-5].

Minimal surfaces are surfaces that locally minimize their area. They refer to the fixed boundary curve of a surface area that is minimal with respect to other surfaces with the same boundary. They are characterized with vanishing mean curvature [6-8].
In light of the existing studies, several researchers were interested in the study of ruled surfaces with Darboux frame. Indeed, in [9], we constructed the ruled surface whose rulings are constant linear combinations of Darboux frame vectors of a regular curve lying on a regular surface of reference in $E^{3}$. We made a comparative study between the two surfaces (the regular surface of reference and the new constructed ruled surface) along their common curve. We investigated properties of the constructed ruled surface and gave examples with illustrations. Furthermore, in [10], the authors showed that the rulings directors of any ruled surface can be expressed with the Darboux frame vectors in $E^{3}$. Besides, they studied characteristic properties of that ruled surface and gave the relationship between Darboux frame and Frenet frame. Moreover, in [11], the authors defined the evolute offsets of ruled surface with Darboux frame and studied its characteristic properties in $E^{3}$.

In curve theory, Smarandache curves were introduced for the first time in Minkowski space time [12]. It defines curves whose position vectors are composed by Frenet-Serret vectors on another regular curve. In [13], Smarandache curves according to Darboux frame were introduced in $E^{3}$. It is a paper that represents an inspiration source for us to make the present work of ruled surface with Smarandache curves according to Darboux frame. 
This paper introduces three ruled surfaces generated by $\mathrm{Tg}$-Smarandache curve, Tn-Smarandache curve, and $g n$-Smarandache curve, according to Darboux frame in $E^{3}$. Theorems giving necessary and sufficient conditions for those surfaces to be developable and minimal are investigated. Finally, an illustrative example is given.

The main contribution of our study is to introduce original notions of ruled surfaces that combine both notions of Smarandache curves and Darboux frame. Our main objective is to study developability and minimalist conditions of such surfaces. Our approach brings together an important significant value in theory and practical application. Indeed, the constructed form of those special ruled surfaces can be useful in applications to medical science, engineering, surface modeling, computer-aided geometric design, computer-aided manufacturing, and computer graphics. In addition, the developability and minimalist properties are the most important properties that are greatly needed and useful in contemporary architecture and architectural design. Hence, the concept of our study opens new avenues for software engineers and architects to some applications in their research fields. Here are some possibilities:

(i) Developing language of geometrical communication for architecture and engineering.

(ii) Constructing algorithms for control point of approximating surfaces.

(iii) Facilitating reconstruction of special developable and minimal surfaces.

(iv) Constructing algorithms for constructing special developable ruled surfaces.

The outline of the paper is structured in the following manner. Some basic notions of ruled surface properties, Darboux frame, and Smarandache curves according to Darboux frame are presented in Section 2. In Section 3, the notions of new three Smarandache ruled surfaces according to Darboux frame are introduced, characterized in terms of developability and minimalist properties, and illustrated in an example. Finally, a summarized conclusion is given in Section 4.

\section{Preliminaries}

In the Euclidean 3 -space $E^{3}$, we consider the usual metric given by

$$
\langle,\rangle=\mathrm{d} x_{1}+\mathrm{d} x_{2}+\mathrm{d} x_{3},
$$

where $\left(x_{1}, x_{2}, x_{3}\right)$ is a rectangular coordinate system of $E^{3}$.

Let $\Psi:(s, v) \in I \times \mathbb{R} \longmapsto c(s)+v \vec{X}(s)$ be a ruled surface in $E^{3}$. It is said to be non-cylindrical, if its rulings directors are not constant, i.e., $\left\|\vec{X}^{\prime}\right\| \neq 0$.

Let us denote by $\vec{m}=\vec{m}(s, v)$ the unit normal on the ruled surface $\Psi$ at a regular point, and we have

$$
\vec{m}=\frac{\Psi_{s} \wedge \Psi_{v}}{\left\|\Psi_{s} \wedge \Psi_{v}\right\|}=\frac{\left(c^{\prime}+v \vec{X}^{\prime}\right) \times \vec{X}}{\left\|\left(c^{\prime}+v \vec{X}^{\prime}\right) \times \vec{X}\right\|},
$$

where $\Psi_{s}=(\partial \Psi(s, v) / \partial s)$ and $\Psi_{v}=(\partial \Psi(s, v) / \partial v)$.

Definition 1 (see [14]). The distribution parameter $\lambda=\lambda(s)$ of a non-cylindrical ruled surface is given by

$$
\lambda=\frac{\operatorname{det}\left(c^{\prime}, \vec{X}, \vec{X}^{\prime}\right)}{\left\|\vec{X}^{\prime}\right\|^{2}} .
$$

Definition 2 (see [14]). A ruled surface is developable if its distribution parameter vanishes.

The first, I, and the second, II, fundamental forms of ruled surface $\Psi$ at a regular point are defined, respectively, by

$$
\begin{gathered}
\mathrm{I}\left(\Psi_{s} \mathrm{~d} s+\Psi_{v} \mathrm{~d} v\right)=E \mathrm{~d} s^{2}+2 F \mathrm{~d} s \mathrm{~d} v+G \mathrm{~d} v^{2}, \\
\mathrm{II}\left(\Psi_{s} \mathrm{~d} s+\Psi_{v} \mathrm{~d} v\right)=e \mathrm{~d} s^{2}+2 f \mathrm{~d} s \mathrm{~d} v+g \mathrm{~d} v^{2},
\end{gathered}
$$

where

$$
\begin{aligned}
& E=\left\|\Psi_{s}\right\|^{2}, F=\left\langle\Psi_{s}, \Psi_{v}\right\rangle, G=\left\|\Psi_{v}\right\|^{2}, \\
& e=\left\langle\Psi_{s s}, \vec{m}\right\rangle, f=\left\langle\Psi_{s v}, \vec{m}\right\rangle, g=\left\langle\Psi_{v v}, \vec{m}\right\rangle=0 .
\end{aligned}
$$

Definition 3. The Gaussian curvature $K$ and the mean curvature $H$ of the ruled surface $\Psi$ at a regular point are given, respectively, by

$$
\begin{aligned}
& K=-\frac{f^{2}}{E G-F^{2}}, \\
& H=\frac{G e-2 F f}{2\left(E G-F^{2}\right)} .
\end{aligned}
$$

Proposition 1 (see [14]). A ruled surface is developable if and only if its Gaussian curvature vanishes.

Proposition 2 (see [14]). A regular surface is minimal if and only if its mean curvature vanishes.

Due to a unit speed curve $\alpha=\alpha(s)$ that lies on a regular surface $\phi=\phi(u, r)$, there exists the Darboux frame and it is denoted by $\{\vec{T}(s), \vec{g}(s), \vec{n}(s)\}$, where $\vec{T}(s)=\alpha \prime(s)$ is the unit tangent vector of the curve $\alpha=\alpha(s)$, $\vec{n}(s)=\left(\phi_{u} \times \phi_{r} /\left\|\phi_{u} \times \phi_{r}\right\|\right)(u(s), r(s))$ is the unit normal vector of the surface $\phi=\phi(u, r)$ along $\alpha=\alpha(s)$, and $\vec{g}(s)$ is the unit vector which is defined by $\vec{g}(s)=\vec{n}(s) \times \vec{T}(s)$.

The derivative formula of the Darboux frame is given as follows: 


$$
\left[\begin{array}{c}
\vec{T}^{\prime} \\
\vec{g}^{\prime} \\
\vec{n}^{\prime}
\end{array}\right]=\left[\begin{array}{ccc}
0 & \rho_{g} & \rho_{n} \\
-\rho_{g} & 0 & \theta_{g} \\
-\rho_{n} & -\theta_{g} & 0
\end{array}\right]\left[\begin{array}{c}
\vec{T} \\
\vec{g} \\
\vec{n}
\end{array}\right]
$$

where $\rho_{g}$ is the geodesic curvature, $\rho_{n}$ is the normal curvature, and $\theta_{g}$ is the geodesic torsion of the curve $\alpha=\alpha(s)$ on the surface $\phi=\phi(u, r)$.

(i) $\alpha(s)$ is a geodesic curve on $\phi$ if its geodesic curvature $\rho_{g}$ vanishes.

(ii) $\alpha(s)$ is an asymptotic line on $\phi$ if its normal curvature $\rho_{n}$ vanishes.

(iii) $\alpha(s)$ is a principal line on $\phi$ if its geodesic torsion $\theta_{g}$ vanishes.

Definition 4 (see [13]). Tg-Smarandache curves according to Darboux frame of the curve $\alpha=\alpha(s)$ on the surface $\phi$ are given by

$$
{ }^{1} \alpha\left(s^{*}(s)\right)=\frac{1}{\sqrt{2}}(\vec{T}(s)+\vec{g}(s)) .
$$

Definition 5 (see [13]). Tn-Smarandache curves according to Darboux frame of the curve $\alpha=\alpha(s)$ on the surface $\phi$ are given by

$$
{ }^{2} \alpha\left(s^{*}(s)\right)=\frac{1}{\sqrt{2}}(\vec{T}(s)+\vec{n}(s)) .
$$

Definition 6 (see [13]). gn-Smarandache curves according to Darboux frame of the curve $\alpha=\alpha(s)$ on the surface $\phi$ are given by

$$
{ }^{3} \alpha\left(s^{*}(s)\right)=\frac{1}{\sqrt{2}}(\vec{g}(s)+\vec{n}(s)) .
$$

\section{Smarandache Ruled Surfaces according to Darboux Frame in $E^{3}$}

We start our section by giving original definitions of Smarandache ruled surfaces according to Darboux frame of a regular curve lying on a regular surface in $E^{3}$.

Definition 7. Let $\alpha=\alpha(s)$ be the $C^{2}$-class differentiable unit speed curve lying on the regular surface $\phi$ in $E^{3}$. Let us denote by $\{\vec{T}(s), \vec{g}(s), \vec{n}(s)\}$ the Darboux frame of $\alpha=$ $\alpha(s)$ on $\phi$. The ruled surfaces generated by Smarandache curves according to Darboux frame of $\alpha=\alpha(s)$ on $\phi$ are as follows:

$$
\left\{\begin{array}{l}
{ }^{1} \omega(s, v)=\frac{1}{\sqrt{2}}(\vec{T}(s)+\vec{g}(s))+v \vec{n}(s), \\
{ }^{2} \omega(s, v)=\frac{1}{\sqrt{2}}(\vec{T}(s)+\vec{n}(s))+v \vec{g}(s), \\
{ }^{3} \omega(s, v)=\frac{1}{\sqrt{2}}(\vec{g}(s)+\vec{n}(s))+v \vec{T}(s) .
\end{array}\right.
$$

These ruled surfaces are called $\mathrm{Tg}$-Smarandache ruled surface, Tn-Smarandache ruled surface, and $g n$-Smarandache ruled surface, according to Darboux frame of the curve $\alpha=\alpha(s)$ on the surface $\phi$, respectively.

In the following, we investigate theorems that give necessary and sufficient conditions for Smarandache ruled surfaces (12) to be developable and minimal.

Theorem 1. Tg-Smarandache ruled surface of (12) is developable if and only if $\alpha(s)$ is a geodesic curve on the surface $\phi$ or $\rho_{n}+\theta_{g}=0$.

Theorem 2. Tg-Smarandache ruled surface of (12) is minimal if and only if $\left(\rho_{g}-v \sqrt{2} \theta_{g}\right)\left[\rho_{g}^{\prime}+\rho_{g}^{2}+\rho_{n}\left(\rho_{n}+\theta_{g}\right)+\right.$ $\left.v \sqrt{2}\left(\rho_{n}^{\prime}-\rho_{g} \theta_{g}\right)\right]-\left(\rho_{g}+v \sqrt{2} \rho_{n}\right)\left[\rho_{g}^{\prime}-\rho_{g}^{2}-\theta_{g}\left(\rho_{n}+\theta_{g}\right)-\right.$ $\left.v \sqrt{2}\left(\theta_{g}^{\prime}+\rho_{g} \rho_{n}\right)\right]-2 \rho_{g}\left(\rho_{n}+\theta_{g}\right)^{2}=0$.

Proof. Differentiating the first line of (12) with respect to $s$ and $v$, respectively, and using (8), we get

$$
\left\{\begin{array}{l}
{ }^{1} \omega_{s}=-\left(\frac{\rho_{g}}{\sqrt{2}}+v \rho_{n}\right) \vec{T}+\left(\frac{\rho_{g}}{\sqrt{2}}-v \theta_{g}\right) \vec{g}+\left(\frac{\rho_{n}+\theta_{g}}{\sqrt{2}}\right) \vec{n}, \\
{ }^{1} \omega_{s}=\vec{n},
\end{array}\right.
$$

The cross product of these two vectors gives the normal vector on $\mathrm{Tg}$-Smarandache ruled surface of (12):

$$
{ }^{1} \omega_{s} \times{ }^{1} \omega_{v}=\left(\frac{\rho_{g}}{\sqrt{2}}-v \theta_{g}\right) \vec{T}+\left(\frac{\rho_{g}}{\sqrt{2}}+v \rho_{n}\right) \vec{g} .
$$

So, under regularity condition, the unit normal takes the following form:

$$
\frac{{ }^{1} \omega_{s} \times{ }^{1} \omega_{v}}{\left\|{ }^{1} \omega_{s} \times{ }^{1} \omega_{v}\right\|}=\frac{1}{\sqrt{\left(\rho_{g}-v \sqrt{2} \theta_{g}\right)^{2}+\left(\rho_{g}+v \sqrt{2} \rho_{n}\right)^{2}}}\left[\left(\rho_{g}-v \sqrt{2} \theta_{g}\right) \vec{T}+\left(\rho_{g}+v \sqrt{2} \rho_{n}\right) \vec{g}\right] .
$$


Applying the norms and the scalar product for (13), we get the components of the first fundamental form of $\mathrm{Tg}$-Smarandache ruled surface of (12), as follows:

$$
\left\{\begin{array}{l}
{ }^{1} E=\frac{\left(\rho_{n}+\theta_{g}\right)^{2}+\left(\rho_{g}+v \sqrt{2} \rho_{n}\right)^{2}+\left(\rho_{g}-v \sqrt{2} \theta_{g}\right)^{2}}{2} \\
{ }^{1} F=\frac{\rho_{n}+\theta_{g}}{\sqrt{2}} \\
{ }^{1} G=1 .
\end{array}\right.
$$

Differentiating (13) with respect to $s$ and $v$, respectively, and using (8), we get

$$
\left\{\begin{array}{l}
{ }^{1} \omega_{s s}=-\left[\left(\frac{\rho_{g}^{\prime}}{\sqrt{2}}+v \rho_{n}^{\prime}\right)+\rho_{g}\left(\frac{\rho_{g}}{\sqrt{2}}-v \theta_{g}\right)+\rho_{n}\left(\frac{\rho_{n}+\theta_{g}}{\sqrt{2}}\right)\right] \vec{T} \\
+\left[\left(\frac{\rho_{g}^{\prime}}{\sqrt{2}}-v \theta_{g}^{\prime}\right)-\rho_{g}\left(\frac{\rho_{g}}{\sqrt{2}}+v \rho_{n}\right)-\theta_{g}\left(\frac{\rho_{n}+\theta_{g}}{\sqrt{2}}\right)\right] \vec{g} \\
+\left[\left(\frac{\rho_{n}^{\prime}+\theta_{g}^{\prime}}{\sqrt{2}}\right)-\rho_{n}\left(\frac{\rho_{g}}{\sqrt{2}}+v \rho_{n}\right)+\theta_{g}\left(\frac{\rho_{g}}{\sqrt{2}}-v \theta_{g}\right)\right] \vec{n}, \\
{ }^{1} \omega_{v s}=-\rho_{n} \vec{T}-\theta_{g} \vec{g} \\
{ }^{1} \omega_{v v}=0 .
\end{array}\right.
$$$$
\left\{\begin{array}{l}
{ }^{1} e=-\frac{\left(\rho_{g}-v \sqrt{2} \theta_{g}\right)\left[\rho_{g}^{\prime}+\rho_{g}^{2}+\rho_{n}\left(\rho_{n}+\theta_{g}\right) / \sqrt{2}+v\left(\rho_{n}^{\prime}-\rho_{g} \theta_{g}\right)\right]}{\sqrt{\left(\rho_{g}-v \sqrt{2} \theta_{g}\right)^{2}+\left(\rho_{g}+v \sqrt{2} \rho_{n}\right)^{2}}} \\
+\frac{\left(\rho_{g}+v \sqrt{2} \rho_{n}\right)\left[\rho_{g}^{\prime}-\rho_{g}^{2}-\theta_{g}\left(\rho_{n}+\theta_{g}\right) / \sqrt{2}-v\left(\theta_{g}^{\prime}+\rho_{g} \rho_{n}\right)\right]}{\sqrt{\left(\rho_{g}-v \sqrt{2} \theta_{g}\right)^{2}+\left(\rho_{g}+v \sqrt{2} \rho_{n}\right)^{2}}}, \\
{ }^{1} f=-\frac{\rho_{g}\left(\rho_{n}+\theta_{g}\right)}{\sqrt{\left(\rho_{g}-v \sqrt{2} \theta_{g}\right)^{2}+\left(\rho_{g}+v \sqrt{2} \rho_{n}\right)^{2}}}, \\
{ }^{1} g=0 .
\end{array}\right.
$$

Consequently, from (16) and (18), we obtain the Gaussian curvature and the mean curvature of $\mathrm{Tg}$-Smarandache ruled surface of (12):

Hence, from (15) and (17), we get the components of the second fundamental form of $\mathrm{Tg}$-Smarandache ruled surface of (12), as follows:

$$
\left\{\begin{array}{l}
{ }^{1} K=-\frac{2 \rho_{g}^{2}\left(\rho_{n}+\theta_{g}\right)^{2}}{\left(\left(\rho_{g}-v \sqrt{2} \theta_{g}\right)^{2}+\left(\rho_{g}+v \sqrt{2} \rho_{n}\right)^{2}\right)^{2}}, \\
{ }^{1} H=-\frac{\left(\rho_{g}-v \sqrt{2} \theta_{g}\right)\left[\rho_{g}^{\prime}+\rho_{g}^{2}+\rho_{n}\left(\rho_{n}+\theta_{g}\right)+v \sqrt{2}\left(\rho_{n}^{\prime}-\rho_{g} \theta_{g}\right)\right]}{\sqrt{2}\left(\left(\rho_{g}-v \sqrt{2} \theta_{g}\right)^{2}+\left(\rho_{g}+v \sqrt{2} \rho_{n}\right)^{2}\right)^{(3 / 2)}} \\
+\frac{\left(\rho_{g}+v \sqrt{2} \rho_{n}\right)\left[\rho_{g}^{\prime}-\rho_{g}^{2}-\theta_{g}\left(\rho_{n}+\theta_{g}\right)-v \sqrt{2}\left(\theta_{g}^{\prime}+\rho_{g} \rho_{n}\right)\right]}{2 \rho_{g}\left(\rho_{n}+\theta_{g}\right)^{2}} \\
\sqrt{2}\left(\left(\rho_{g}-v \sqrt{2} \theta_{g}\right)^{2}+\left(\rho_{g}+v \sqrt{2} \rho_{n}\right)^{2}\right)^{(3 / 2)}
\end{array}\right.
$$

which replies to both above theorems.

Corollary 1. If $\alpha(s)$ is a geodesic curve on the surface $\phi$, then (i) Tg-Smarandache ruled surface is developable.

(ii) Tg-Smarandache ruled surface is minimal if and only if $\rho_{n}^{\prime} \theta_{g}-\rho_{n} \theta_{g}^{\prime}=0$. 
Theorem 3. Tn-Smarandache ruled surface of (12) is developable if and only if $c(s)$ is an asymptotic line on the surface $\phi$ or $\theta_{g}=\rho_{g}$.

Theorem 4. Tn-Smarandache ruled surface of (12) is minimal if and only if $\left(\rho_{n}+v \sqrt{2} \theta_{g}\right)\left[\rho_{n}^{\prime}+\rho_{n}^{2}+\rho_{g}\left(\rho_{g}-\theta_{g}\right)+\right.$ $\left.v \sqrt{2}\left(\rho_{g}^{\prime}+\rho_{n} \theta_{g}\right)\right]-\left(\rho_{n}+v \sqrt{2} \rho_{g}\right)\left[\rho_{n}^{\prime}-\rho_{n}^{2}+\theta_{g}\left(\rho_{g}-\theta_{g}\right)+\right.$ $\left.v \sqrt{2}\left(\theta_{g}^{\prime}-\rho_{n} \rho_{g}\right)\right]-2 \rho_{n}\left(\theta_{g}-\rho_{g}\right)^{2}=0$.

Proof. Differentiating the second line of (12) with respect to $s$ and $v$, respectively, and using (8), we get

$$
\left\{\begin{array}{l}
{ }^{1} \omega_{s}=-\left(\frac{\rho_{n}}{\sqrt{2}}+v \rho_{g}\right) \vec{T}+\left(\frac{\rho_{g}-\theta_{g}}{\sqrt{2}}\right) \vec{g}+\left(\frac{\rho_{n}}{\sqrt{2}}+v \theta_{g}\right) \vec{n}, \\
{ }^{1} \omega_{v}=\vec{g},
\end{array}\right.
$$

which implies that the normal vector on $T n$-Smarandache ruled surface of (12) is

$$
{ }^{2} \omega_{s} \times{ }^{2} \omega_{v}=-\left(\frac{\rho_{n}}{\sqrt{2}}+v \theta_{g}\right) \vec{T}-\left(\frac{\rho_{n}}{\sqrt{2}}+v \rho_{g}\right) \vec{n} .
$$
by

So, under regularity condition, the unit normal is given

$$
\frac{{ }^{2} \omega_{s} \times{ }^{2} \omega_{v}}{\left\|{ }^{2} \omega_{s} \times{ }^{2} \omega_{v}\right\|}=-\frac{1}{\sqrt{\left(\rho_{n}+v \sqrt{2} \theta_{g}\right)^{2}+\left(\rho_{n}+v \sqrt{2} \rho_{g}\right)^{2}}}\left[\left(\rho_{n}+v \sqrt{2} \theta_{g}\right) \vec{T}+\left(\rho_{n}+v \sqrt{2} \rho_{g}\right) \vec{n}\right]
$$

Applying the norms and the scalar product for (20), we get the components of the first fundamental form of Tn-Smarandache ruled surface of (12):

$$
\left\{\begin{array}{l}
{ }^{2} E=\frac{\left(\rho_{g}-\theta_{g}\right)^{2}+\left(\rho_{n}+v \sqrt{2} \rho_{g}\right)^{2}+\left(\rho_{n}+v \sqrt{2} \theta_{g}\right)^{2}}{2} \\
{ }^{2} F=\frac{\rho_{g}-\theta_{g}}{\sqrt{2}} \\
{ }^{2} G=1 .
\end{array}\right.
$$

Differentiating (20) with respect to $s$ and $v$, respectively, and using (8), we get 


$$
\left\{\begin{array}{l}
{ }^{2} e=\frac{\left(\rho_{n}+v \sqrt{2} \theta_{g}\right)\left[\rho_{n}^{\prime}+\rho_{n}^{2}+\rho_{g}\left(\rho_{g}-\theta_{g}\right) / \sqrt{2}+v\left(\rho_{g}^{\prime}+\rho_{n} \theta_{g}\right)\right]}{\sqrt{\left(\rho_{n}+v \sqrt{2} \theta_{g}\right)^{2}+\left(\rho_{n}+v \sqrt{2} \rho_{g}\right)^{2}}} \\
-\frac{\left(\rho_{n}+v \sqrt{2} \rho_{g}\right)\left[\rho_{n}^{\prime}-\rho_{n}^{2}+\theta_{g}\left(\rho_{g}-\theta_{g}\right) / \sqrt{2}+v\left(\theta_{g}^{\prime}-\rho_{n} \rho_{g}\right)\right]}{\sqrt{\left(\rho_{n}+v \sqrt{2} \theta_{g}\right)^{2}+\left(\rho_{n}+v \sqrt{2} \rho_{g}\right)^{2}}}, \\
{ }^{2} f=-\frac{\rho_{n}\left(\theta_{g}-\rho_{g}\right)}{\sqrt{\left(\rho_{n}+v \sqrt{2} \theta_{g}\right)^{2}+\left(\rho_{n}+v \sqrt{2} \rho_{g}\right)^{2}}}, \\
{ }^{2} g=0 .
\end{array}\right.
$$

Thus, from (23) and (25), we obtain the Gaussian curvature and the mean curvature of $T n$-Smarandache ruled surface of (12):

$$
\left\{\begin{array}{l}
{ }^{2} K=-\frac{2 \rho_{n}^{2}\left(\theta_{g}-\rho_{g}\right)^{2}}{\left[\left(\rho_{n}+v \sqrt{2} \theta_{g}\right)^{2}+\left(\rho_{n}+v \sqrt{2} \rho_{g}\right)^{2}\right]^{(3 / 2)}}, \\
{ }^{2} H=\frac{\left(\rho_{n}+v \sqrt{2} \theta_{g}\right)\left[\rho_{n}^{\prime}+\rho_{n}^{2}+\rho_{g}\left(\rho_{g}-\theta_{g}\right)+v \sqrt{2}\left(\rho_{g}^{\prime}+\rho_{n} \theta_{g}\right)\right]}{\sqrt{2}\left(\left(\rho_{n}+v \sqrt{2} \theta_{g}\right)^{2}+\left(\rho_{n}+v \sqrt{2} \rho_{g}\right)^{2}\right)^{(3 / 2)}} \\
-\frac{\left(\rho_{n}+v \sqrt{2} \rho_{g}\right)\left[\rho_{n}^{\prime}-\rho_{n}^{2}+\theta_{g}\left(\rho_{g}-\theta_{g}\right)+v \sqrt{2}\left(\theta_{g}^{\prime}-\rho_{n} \rho_{g}\right)\right]}{\sqrt{2}\left(\left(\rho_{n}+v \sqrt{2} \theta_{g}\right)^{2}+\left(\rho_{n}+v \sqrt{2} \rho_{g}\right)^{2}\right)^{(3 / 2)}} \\
-\frac{2 \rho_{n}\left(\theta_{g}-\rho_{g}\right)^{2}}{\sqrt{2}\left(\left(\rho_{n}+v \sqrt{2} \theta_{g}\right)^{2}+\left(\rho_{n}+v \sqrt{2} \rho_{g}\right)^{2}\right)^{(3 / 2)}},
\end{array}\right.
$$

which replies to both above theorems.

Corollary 2. If $\alpha(s)$ is an asymptotic line on the surface $\phi$, then

(i) Tn-Smarandache ruled surface is developable.

(ii) Tn-Smarandache ruled surface is minimal if and only if $\rho_{g}^{\prime} \theta_{g}-\rho_{g} \theta_{g}^{\prime}=0$.

Theorem 5. gn-Smarandache ruled surface of (12) is developable if and only if $\alpha(s)$ is a principal line on the surface $\phi$ or $\rho_{g}+\rho_{n}=0$.

Theorem 6. gn-Smarandache ruled surface of (12) is minimal if and only if $\left(\theta_{g}+v \sqrt{2} \rho_{n}\right)\left[-\theta_{g}^{\prime}-\rho_{g}\left(\rho_{g}+\rho_{n}\right)-\theta_{g}^{2}+\right.$ $\left.v \sqrt{2}\left(\rho_{g}^{\prime}-\rho_{n} \theta_{g}\right)\right]-\left(-\theta_{g}+v \sqrt{2} \rho_{g}\right)\left[\theta_{g}^{\prime}-\rho_{n}\left(\rho_{g}+\rho_{n}\right)-\theta_{g}^{2}+\right.$ $\left.v \sqrt{2}\left(\rho_{n}^{\prime}+\rho_{g} \theta_{g}\right)\right]+2 \theta_{g}\left(\rho_{g}+\rho_{n}\right)^{2}=0$.
Proof. Differentiating the third line of (12) with respect to $s$ and $v$, respectively, and using (8), we get

$$
\left\{\begin{array}{l}
{ }^{3} \omega_{s}=-\left(\frac{\rho_{g}+\rho_{n}}{\sqrt{2}}\right) \vec{T}+\left(-\frac{\theta_{g}}{\sqrt{2}}+v \rho_{g}\right) \vec{g}+\left(\frac{\theta_{g}}{\sqrt{2}}+v \rho_{n}\right) \vec{n}, \\
{ }^{3} \omega_{v}=\vec{T}
\end{array}\right.
$$

By realizing the cross product of both vectors of (27), we get the normal vector on $g n$-Smarandache ruled surface of (12):

$$
{ }^{3} \omega_{s} \times{ }^{3} \omega_{v}=\left(\frac{\theta_{g}}{\sqrt{2}}+v \rho_{n}\right) \vec{g}-\left(-\frac{\theta_{g}}{\sqrt{2}}+v \rho_{g}\right) \vec{n} .
$$

So, under regularity condition, the unit normal takes the following form: 


$$
\frac{{ }^{3} \omega_{s} \times{ }^{3} \omega_{v}}{\left\|{ }^{3} \omega_{s} \times{ }^{3} \omega_{v}\right\|}=\frac{1}{\sqrt{\left(\theta_{g}+v \sqrt{2} \rho_{n}\right)^{2}+\left(-\theta_{g}+v \sqrt{2} \rho_{g}\right)^{2}}}\left[\left(\theta_{g}+v \sqrt{2} \rho_{n}\right) \vec{g}-\left(-\theta_{g}+v \sqrt{2} \rho_{g}\right) \vec{n}\right]
$$

Applying the norms and the scalar product for (27), we get the components of the first fundamental form of gn-Smarandache ruled surface of (12), as follows:

$$
\left\{\begin{array}{l}
{ }^{3} E=\frac{\left(\rho_{g}+\rho_{n}\right)^{2}+\left(-\theta_{g}+v \sqrt{2} \rho_{g}\right)^{2}+\left(\theta_{g}+v \sqrt{2} \rho_{n}\right)^{2}}{2} \\
{ }^{3} F=-\frac{\rho_{g}+\rho_{n}}{\sqrt{2}} \\
{ }^{3} G=1
\end{array}\right.
$$

Differentiating (27) with respect to $s$ and $v$, respectively, and using (8), we get

$$
\left\{\begin{array}{l}
{ }^{3} \omega_{s s}=-\left[\left(\frac{\rho_{g}^{\prime}+\rho_{n}^{\prime}}{\sqrt{2}}\right)+\rho_{g}\left(-\frac{\theta_{g}}{\sqrt{2}}+v \rho_{g}\right)+\rho_{n}\left(\frac{\theta_{g}}{\sqrt{2}}+v \rho_{n}\right)\right] \vec{T} \\
+\left[\left(-\frac{\theta_{g}^{\prime}}{\sqrt{2}}+v \rho_{g}^{\prime}\right)-\rho_{g}\left(\frac{\rho_{g}+\rho_{n}}{\sqrt{2}}\right)-\theta_{g}\left(\frac{\theta_{g}}{\sqrt{2}}+v \rho_{n}\right)\right] \vec{g} \\
+\left[\left(\frac{\theta_{g}^{\prime}}{\sqrt{2}}+v \rho_{n}^{\prime}\right)-\rho_{n}\left(\frac{\rho_{g}+\rho_{n}}{\sqrt{2}}\right)+\theta_{g}\left(-\frac{\theta_{g}}{\sqrt{2}}+v \rho_{g}\right)\right] \vec{n} \\
{ }^{3} \omega_{v s}=\rho_{g} \vec{g}+\rho_{n} \vec{n} \\
{ }^{3} \omega_{v v}=0 .
\end{array}\right.
$$

Hence, from (29) and (31), we get the components of the second fundamental form of $g n$-Smarandache ruled surface of (12), as follows:

$$
\left\{\begin{array}{l}
3 e=\frac{\left(\theta_{g}+v \sqrt{2} \rho_{n}\right)\left[\left(-\left(\theta_{g}^{\prime} / \sqrt{2}\right)+v \rho_{g}^{\prime}\right)-\rho_{g}\left(\left(\rho_{g}+\rho_{n}\right) / \sqrt{2}\right)-\theta_{g}\left(\left(\theta_{g} / \sqrt{2}\right)+v \rho_{n}\right)\right]}{\sqrt{\left(\theta_{g}+v \sqrt{2} \rho_{n}\right)^{2}+\left(-\theta_{g}+v \sqrt{2} \rho_{g}\right)^{2}}} \\
-\frac{\left(-\theta_{g}+v \sqrt{2} \rho_{g}\right)\left[\left(\left(\theta_{g}^{\prime} / \sqrt{2}\right)+v \rho_{n}^{\prime}\right)-\rho_{n}\left(\left(\rho_{g}+\rho_{n}\right) / \sqrt{2}\right)+\theta_{g}\left(-\left(\theta_{g} / \sqrt{2}\right)+v \rho_{g}\right)\right]}{\sqrt{\left(\theta_{g}+v \sqrt{2} \rho_{n}\right)^{2}+\left(-\theta_{g}+v \sqrt{2} \rho_{g}\right)^{2}}} \\
{ }^{3} f=\frac{\theta_{g}\left(\rho_{g}+\rho_{n}\right)}{\sqrt{\left(\theta_{g}+v \sqrt{2} \rho_{n}\right)^{2}+\left(-\theta_{g}+v \sqrt{2} \rho_{g}\right)^{2}}}, \\
{ }^{3} g=0 .
\end{array}\right.
$$




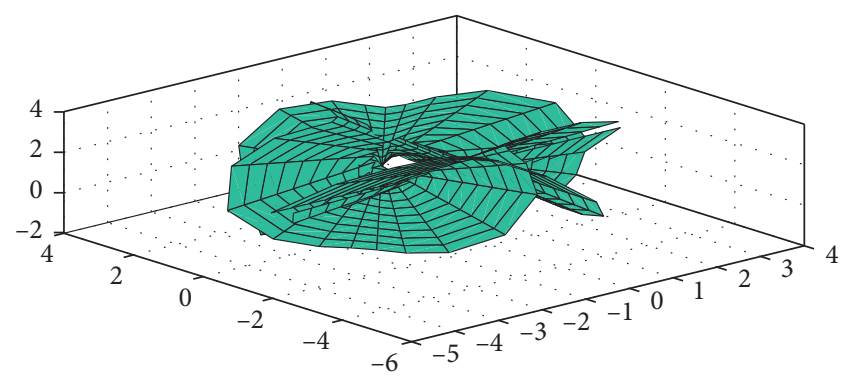

Figure 1: $T g$-Smarandache ruled surface (36).

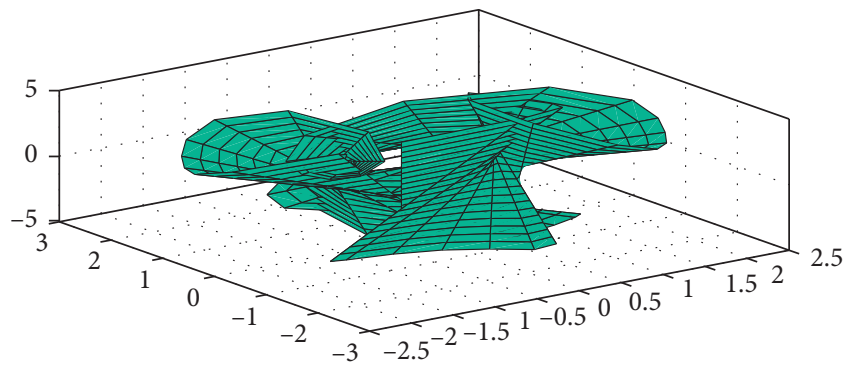

FIgURE 2: Tn-Smarandache ruled surface (37).

Consequently, from (30) and (32), we obtain the Gaussian curvature and the mean curvature of gn-Smarandache ruled surface of (12):

$$
\left\{\begin{array}{l}
{ }^{3} K=-\frac{2 \theta_{g}^{2}\left(\rho_{g}+\rho_{n}\right)^{2}}{\left[\left(\theta_{g}+v \sqrt{2} \rho_{n}\right)^{2}+\left(-\theta_{g}+v \sqrt{2} \rho_{g}\right)^{2}\right]^{2}}, \\
{ }^{3} H=\frac{\left(\theta_{g}+v \sqrt{2} \rho_{n}\right)\left[-\theta_{g}^{\prime}-\rho_{g}\left(\rho_{g}+\rho_{n}\right)-\theta_{g}^{2}+v \sqrt{2}\left(\rho_{g}^{\prime}-\rho_{n} \theta_{g}\right)\right]}{\left(\left(\theta_{g}+v \sqrt{2} \rho_{n}\right)^{2}+\left(-\theta_{g}+v \sqrt{2} \rho_{g}\right)^{2}\right)^{(3 / 2)}} \\
-\frac{\left(-\theta_{g}+v \sqrt{2} \rho_{g}\right)\left[\theta_{g}^{\prime}-\rho_{n}\left(\rho_{g}+\rho_{n}\right)-\theta_{g}^{2}+v \sqrt{2}\left(\rho_{n}^{\prime}+\rho_{g} \theta_{g}\right)\right]}{\left(\left(\theta_{g}+v \sqrt{2} \rho_{n}\right)^{2}+\left(-\theta_{g}+v \sqrt{2} \rho_{g}\right)^{2}\right)^{(3 / 2)}} \\
+\frac{2 \theta_{g}\left(\rho_{g}+\rho_{n}\right)^{2}}{\sqrt{2}\left(\left(\theta_{g}+v \sqrt{2} \rho_{n}\right)^{2}+\left(-\theta_{g}+v \sqrt{2} \rho_{g}\right)\right)^{(3 / 2)}},
\end{array}\right.
$$

which replies to both above theorems.

Corollary 3. If $\alpha(s)$ is a principal line on the surface $\phi$, then

(i) gn-Smarandache ruled surface is developable.

(ii) gn-Smarandache ruled surface is minimal if and only if $\rho_{n}^{\prime} \rho_{g}-\rho_{n} \rho_{g}^{\prime}=0$.

Now, we will give an example for the whole study and present corresponding illustrations.

Example 1. Let us consider the unit speed curve ${ }^{e} \alpha={ }^{e} \alpha(s)$ and the regular surface ${ }^{e} \phi={ }^{e} \phi(u, r)$ defined, respectively, by

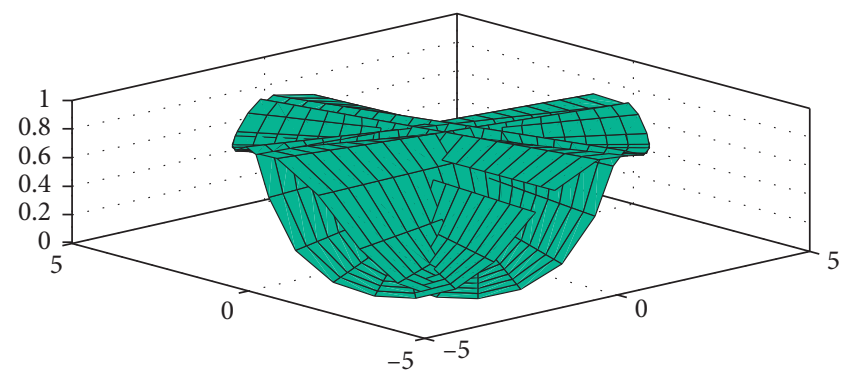

Figure 3: gn-Smarandache ruled surface (38).

$$
\begin{aligned}
{ }^{e} \alpha(s) & =\left(2 \cos \left(\frac{s}{2}\right), 2 \sin \left(\frac{s}{2}\right), 0\right), \\
{ }^{e} \varphi(u, r) & =\left(2 \cos \left(\frac{u}{2}\right)-\frac{r}{\sqrt{2}} \sin \left(\frac{u}{2}\right), 2 \sin \frac{u}{2}-\frac{r}{\sqrt{2}} \cos \left(\frac{u}{2}\right), \frac{r}{\sqrt{2}}\right) .
\end{aligned}
$$

It is clear that ${ }^{e} \alpha={ }^{e} \alpha(s)$ lies on $e \phi={ }^{e} \phi(u, r)$; indeed, ${ }^{e} \alpha(s)={ }^{e} \varphi(s, 0)$. The Darboux frame of ${ }^{e} \alpha={ }^{e} \alpha(s)$ on ${ }^{e} \phi=$ ${ }^{e} \phi(u, r)$ is given by

$$
\vec{T}(s)=\left(\begin{array}{c}
-\sin \left(\frac{s}{2}\right) \\
\cos \left(\frac{s}{2}\right) \\
0
\end{array}\right)
$$

$$
\vec{g}(s)=\frac{1}{\sqrt{1+\sin ^{2}(s)}}\left(\begin{array}{c}
-\sin (s) \cos \left(\frac{s}{2}\right) \\
-\sin (s) \sin \left(\frac{s}{2}\right) \\
1
\end{array}\right)
$$$$
\vec{n}(s)=\frac{1}{\sqrt{1+\sin ^{2}(s)}}\left(\begin{array}{c}
\cos \left(\frac{s}{2}\right) \\
\sin \left(\frac{s}{2}\right) \\
\sin (s)
\end{array}\right)
$$

$$
\begin{aligned}
& \rho_{n}(s)=\frac{-1}{2 \sqrt{1+\sin ^{2}(s)}}, \\
& \rho_{g}(s)=\frac{\sin (s)}{2 \sqrt{1+\sin ^{2}(s)}}, \\
& \theta_{g}(s)=-\frac{\cos (s)}{1+\sin ^{2}(s)} .
\end{aligned}
$$

Consequently, $\mathrm{Tg}$-Smarandache ruled surface, $T n$-Smarandache ruled surface, and $g n$-Smarandache ruled 
surface, according to Darboux frame of ${ }^{e} \alpha={ }^{e} \alpha(s)$ on ${ }^{e} \phi={ }^{e} \phi(u, r)$, are given with their illustrations (Figures 1-3), respectively, as follows:

$$
\begin{aligned}
& 1 \omega(s, v)=\frac{1}{\sqrt{2}}\left(\begin{array}{c}
-\sin \left(\frac{s}{2}\right)-\frac{\sin (s) \cos (s / 2)}{\sqrt{1+\sin ^{2}(s)}} \\
\cos \left(\frac{s}{2}\right)-\frac{\sin (s) \sin (s / 2)}{\sqrt{1+\sin ^{2}(s)}} \\
\sqrt{1+\sin ^{2}(s)}
\end{array}\right)+\frac{v}{\sqrt{1+\sin ^{2}(s)}}\left(\begin{array}{c}
\cos \left(\frac{s}{2}\right) \\
\sin \left(\frac{s}{2}\right) \\
\sin (s)
\end{array}\right) \\
& { }^{2} \omega(s, v)=\frac{1}{\sqrt{2}}\left(\begin{array}{c}
-\sin \left(\frac{s}{2}\right)+\frac{\cos (s / 2)}{\sqrt{1+\sin ^{2}(s)}} \\
\cos \left(\frac{s}{2}\right)+\frac{\sin (s / 2)}{\sqrt{1+\sin ^{2}(s)}} \\
\frac{\sin (s)}{\sqrt{1+\sin ^{2}(s)}}
\end{array}\right)+\frac{v}{\sqrt{1+\sin ^{2}(s)}}\left(\begin{array}{c}
-\sin (s) \cos \left(\frac{s}{2}\right) \\
-\sin (s) \sin \left(\frac{s}{2}\right) \\
1
\end{array}\right) \\
& { }^{3} \omega(s, v)=\frac{1}{\sqrt{2\left(1+\sin ^{2}(s)\right)}}\left(\begin{array}{c}
-\sin (s) \cos \left(\frac{s}{2}\right)+\cos \left(\frac{s}{2}\right) \\
-\sin (s) \sin \left(\frac{s}{2}\right)+\sin \left(\frac{s}{2}\right) \\
1+\sin (s)
\end{array}\right)+v\left(\begin{array}{c}
-\sin \left(\frac{s}{2}\right) \\
\cos \left(\frac{s}{2}\right) \\
0
\end{array}\right) .
\end{aligned}
$$

\section{Conclusion}

In this study, we proposed a new approach of constructing three special ruled surfaces inspired by Smarandache curves according to Darboux frame. It was about $\mathrm{Tg}$-Smarandache ruled surface, $\mathrm{Tn}$-Smarandache ruled surface, and gn-Smarandache ruled surface according to Darboux frame of a unit speed curve $\alpha(s)$ lying on an arbitrary regular surface $\phi$. We reached our objective by investigating necessary and sufficient conditions for those surfaces to be developable and minimal. We found that the results depend on the properties of the geodesic curvature, the normal curvature, and the geodesic torsion of the curve $\alpha(s)$ on the reference surface $\phi$. Hence, we can anticipate the developability and minimality after making the right choice for the curve $\alpha(s)$ and the surface $\phi$. An example was given with illustrations to see the geometric shapes of such surfaces.

On the other hand, the approach of our study opened up opportunities for scientists in several areas such as surface modeling, engineering, contemporary architecture, and architectural design, to use our results as tools for their future applications.

\section{Data Availability}

No data were used to support this study.

\section{Conflicts of Interest}

The author declares that there are no conflicts of interest.

\section{References}

[1] D. J. Struik, Lectures on Classical Differential Geometry, Addison-Wesley, Dover, NH, USA, 2nd edition, 1988.

[2] M. P. Do Carmo, Differential Geometry of Curves and Surfaces, Prentice Hall, Englewood Cliffs, NJ, USA, 1976.

[3] G. Hu, H. Cao, J. Wu, and G. Wei, "Construction of developable surfaces using generalized C-Bézier bases with shape parameters," Computational and Applied Mathematics, vol. 39, no. 3, p. 157, 2020.

[4] T. G. Nelson, T. Zimmerman, S. P. Magleby, R. J. Lang, and L. L. Howell, "Developable mechanisms on developable surfaces," Science Robotics, vol. 4, no. 27, 2019.

[5] P. Alegre, K. Arslan, A. Carriazo, C. Murathan, and G. Öztürk, "Some special types of developable ruled surface," Hacettepe 
Journal of Mathematics and Statistics, vol. 39, no. 3, pp. 319-325, 2010.

[6] W. Y. Lam, "Minimal surfaces from infinitesimal deformations of circle packings," Advances in Mathematics, vol. 362, Article ID 106939, 2020.

[7] T. H. Colding and C. D. Lellis, "The min-max construction of minimal surfaces," Surveys in Differential Geometry, vol. 8, no. 1, pp. 75-107, 2003.

[8] J. L. M. Barbosa and A. G. Colares, Minimal Surfaces in $\mathbb{R}^{3}$, Springer Verlag, Berlin Heidelberg, Germany, 1986.

[9] S. Ouarab, A. O. Chahdi, and M. Izid, "Ruled surface generated by a curve lying on a regular surface and its characterizations," Journal for Geometry and Graphics, vol. 24, no. 2, pp. $257-267,2020$.

[10] G. Y. Senturk and S. Yuce, "Characteristic proprerties of the ruled surface with Darboux frame," Kuwait Journal of Science, vol. 42, no. 2, pp. 14-33, 2015.

[11] G. Y. Senturk and S. Yuce, "On the evolute offsets of ruled surfaces using the Darboux frame," Communications Faculty of Sciences University of Ankara Series Al Mathematics and Statistics, vol. 68, no. 2, pp. 1256-1264, 2019.

[12] M. Turgut and S. Yilmaz, "Smarandache curves in Minkowski space-time," International Journal of Mathematical Combinatorics, vol. 3, pp. 51-55, 2008.

[13] O. Bektas and S. Yüce, "Special Smarandache curves according to Darboux frame in Euclidean 3- space," Romanian Journal of Mathematics and Computer Science, vol. 3, no. 1, pp. 48-59, 2012.

[14] B. O’Neill, Semi-Riemannian Geometry, Academic Press, New York, NY, USA, 1983. 